\title{
A Sufficient Condition for the Almost Global Stability of Nonlinear Switched Systems with Average Dwell Time
}

\author{
Ferruh İlhan $^{1}$, Özkan Karabacak ${ }^{2 *}$ and Rafael Wisniewski ${ }^{2}$
}

\begin{abstract}
A sufficient condition for the almost global stability of nonlinear switched systems under average dwell time restriction is obtained. This condition is derived leaning upon the existence of multiple Lyapunov densities, which are associated to subsystems and satisfy some compatibility conditions. An upper bound for the average dwell time that ensures almost global stability is obtained.
\end{abstract}

\section{INTRODUCTION}

Solutions of a nonlinear system may be attracting for almost all initial states without exhibiting global asymptotic stability. Such a situation may take place due to the structure of the state space [1] or due to some degenericity in dynamics [2]. This property, that goes by the name almost global stability or almost everywhere stability, has been under consideration of the systems and control community after the introduction of dual Lyapunov theorem by Rantzer [3]. Rantzer's theorem provides a characterization of almost global stability by the existence of a density function, mostly called Lyapunov density. Lyapunov densities have been studied for the analysis of dynamical systems [4], [5], [6], [7], [8], [9], [10], [11], [12], for the design of control systems [13], [2], [14], [15], [16], [17], [18], and for safety verification [19]. Various generalizations of Rantzer's theorem appeared in literature, for example to discrete-time systems [6], to discontinuous systems [20], to smoothly time-varying systems [21], [22] and to stochastic systems [23]. Recently, we have studied the extension of Rantzer's theorem to nonlinear switched systems and obtained sufficient conditions for the almost global stability of nonlinear switched systems based on the existence of multiple Lyapunov densities and a minimum dwell time condition [24].

In this work, we continue with this line of research and obtain a sufficient condition for the almost global stability of nonlinear switched systems with average dwell time. While the dwell time condition in [24] can be characterized by the maximum cycle ratio of a doubly-weighted complete digpraph, the average dwell time condition obtained in this work can be characterized by the maximum cycle mean. Fast algorithms for finding the maximum cycle ratio and maximum cycle mean exist in literature which can be used even if the number of subsystems is large.

This work has been supported by the Independent Research Fund Denmark (DeBaTe)

*Corresponding author

${ }^{1}$ Department of Electrical and Electronics Engineering, Turkish-German University, Beykoz, Istanbul ilhanetau . edu. tr

${ }^{2}$ Department of Electronic Systems, Automation and Control, Aalborg University, Fredrik Bajers Vej 7 C, 9220 Aalborg East, Denmark ozk@es.aau.dk, raf@es.aau.dk
For simplicity, we consider the almost global stability of a common equilibrium. However, same results directly apply if a common invariant set is considered and the Lipschitz condition is assumed in the vicinity of the invariant set.

The technique of proof of the main result is similar to the proof in our earlier work [24] for switched system under dwell time constraints. However, instead of considering discretization and using sufficient conditions for the almost global stability of discrete-time systems, we apply directly a sufficient condition (Lemma 2) for the almost global stability of continuous-time systems. This approach facilitates the derivation of the average dwell condition for the almost global stability.

The organization of the paper is as follows: Section II contains some preliminaries on the almost global stability of autonomous systems and switched systems. Section III states the main result of the paper, which is proven in Section IV using the theory of transfer operators of nonlinear systems, namely the Frobenius-Perron and Koopman operators.

\section{Preliminaries}

\section{A. Almost Global Stability of Autonomous Systems}

We consider the autonomous system

$$
\dot{x}=f(x), \quad x \in \mathbb{R}^{n},
$$

where $f: \mathbb{R}^{n} \rightarrow \mathbb{R}^{n}$ is continuously differentiable and $f(\mathbf{0})=\mathbf{0}$. Almost global stability of (1), namely the convergence of almost all solutions of (1) to $\mathbf{0}$ can be characterized by the following version of Rantzer's theorem.

Proposition 1 ([24]): Assume that there exists a nonnegative, continuously differentiable function $\rho: \mathbb{R}^{n} \backslash\{\mathbf{0}\} \rightarrow$ $\mathbb{R}$ satisfying

$$
\begin{aligned}
& (1+\|f(x)\|) \rho(x) \text { is integrable away from } \mathbf{0}, \\
& \nabla \cdot(\rho f)(x)>0 \text { for almost all } x \in \mathbb{R}^{n} \backslash\{\mathbf{0}\} .
\end{aligned}
$$

Then, for almost every initial state $x_{0} \in \mathbb{R}^{n}$, a forwardcomplete solution $x: \mathbb{R}_{\geq 0} \rightarrow \mathbb{R}^{n}$ of (1) with $x(0)=x_{0}$ exists and converges to $\mathbf{0}$ as $t \rightarrow \infty$.

Proposition 1 is different from Rantzer's original theorem only by the assumption of integrability of $(1+\|f(x)\|) \rho(x)$ replacing the integrability of $\|f(x)\| \rho(x) /\|x\|$ in Rantzer's theorem. We use the former assumption as it implies the integrability of $\rho(x)$, which is used to achieve almost global stability of switched systems. 


\section{B. Almost Global Stability of Nonlinear Switched Systems}

Consider a nonlinear switched system with $D$ subsystems

$$
\dot{x}=f_{\sigma(t)}(x), \quad x \in \mathbb{R}^{n}, \quad \sigma \in \mathcal{S}, \quad t \geq 0,
$$

where $\mathcal{S}$ is a set of admissible switching signals (rightcontinuous, piecewise constant functions) $\sigma:[0, \infty) \rightarrow$ $\{1,2, \ldots, D\}$. We either consider $\mathcal{S}=\mathcal{S}_{\text {dwell }}[\tau]$, the set of switching signals satisfying the dwell time condition

$$
t_{k+1}-t_{k} \geq \tau
$$

where $t_{k}$ is the $k$ 'th switching instant, or we consider $\mathcal{S}=$ $\mathcal{S}_{\text {average }}[\tau]$, the set of switching signals satisfying the average dwell time condition

$$
N(t) \leq N_{0}+\frac{t}{\tau}
$$

where $N(t)$ is the number of switchings before time $t$ and $N_{0}$ is a nonnegative integer called the chattering bound. We assume that each subsystem vector field $f_{i}: \mathbb{R}^{n} \rightarrow \mathbb{R}^{n}$ is continuously differentiable and has an equilibrium at the origin, i.e., $f_{i}(0)=0, i=1, \ldots, D$.

Definition 1 (Almost global stability): We say that the system (2) is almost globally stable if for each switching signal $\sigma \in \mathcal{S}$, a solution of (2) exists for all $t \geq 0$ and converges to $\mathbf{0}$ for almost every initial state.

The following theorem provides a sufficient condition for the almost global stability of (1) for $\mathcal{S}=\mathcal{S}_{\text {dwell }}[\tau]$.

Theorem 1 ([24]): Consider the switched system (1). Suppose that for each $p \in\{1,2, \ldots, D\}$, there exist a constant $\kappa_{p}>0$ and a non-negative, continuously differentiable function $\rho_{p}: \mathbb{R}^{n} \backslash\{\mathbf{0}\} \rightarrow \mathbb{R}$, such that

$$
\begin{gathered}
\left(1+\left\|f_{p}(x)\right\|\right) \rho_{p}(x) \text { is integrable away from } \mathbf{0}, \\
\nabla \cdot\left(\rho_{p} f_{p}\right)(x) \geq \kappa_{p} \rho_{p}(x) \forall x \in \mathbb{R}^{n} \backslash\{\mathbf{0}\} .
\end{gathered}
$$

Suppose also that the functions $\rho_{p}, p \in\{1, \ldots, D\}$ satisfy the following compatibility condition

$$
\begin{gathered}
\forall p, m \in\{1, \ldots, D\}, \exists c_{p m} \in \mathbb{R}_{>0}: \\
\rho_{p}(x) \leq c_{p m} \rho_{m}(x) \forall x \in \mathbb{R}^{n} \backslash\{\mathbf{0}\} .
\end{gathered}
$$

Then, the system (2) for $\mathcal{S}=\mathcal{S}_{\text {dwell }}[\tau]$ is almost globally stable for any

$$
\tau>\tau_{\min }:=\min _{\beta_{1}, \ldots, \beta_{D} \in \mathbb{R}_{>0}} \max _{p, m \in\{1,2, \ldots, D\}} \frac{\ln \left(\frac{\beta_{p}}{\beta_{m}} c_{p m}\right)}{\kappa_{p}} .
$$

Note that the definition $\tau_{\min }$ in (6) is equivalent to the maximum cycle ratio of a doubly weighted complete graph and hence can be computed using the fast algorithms available in literature [25] (see Remark 2 in [24]).

\section{MAin Result}

We now present a sufficient condition for the almost global stability of (2) for $\mathcal{S}=\mathcal{S}_{\text {average }}[\tau]$.

Theorem 2: Consider the switched system (2). Suppose that there exists a constant $\kappa>0$, and for each $p \in$
$\{1,2, \ldots, D\}$, there exists a non-negative, continuously differentiable function $\rho_{p}: \mathbb{R}^{n} \backslash\{\mathbf{0}\} \rightarrow \mathbb{R}$, such that

$$
\begin{gathered}
\left(1+\left\|f_{p}(x)\right\|\right) \rho_{p}(x) \text { is integrable away from } \mathbf{0}, \\
\nabla \cdot\left(\rho_{p} f_{p}\right)(x) \geq \kappa \rho_{p}(x) \forall x \in \mathbb{R}^{n} \backslash\{\mathbf{0}\} .
\end{gathered}
$$

Suppose also that the functions $\rho_{p}, p \in\{1, \ldots, D\}$ satisfy the following compatibility condition

$$
\begin{aligned}
& \forall p, m \in\{1, \ldots, D\}, \exists c_{p m} \in \mathbb{R}_{>0}: \\
& \rho_{p}(x) \leq c_{p m} \rho_{m}(x) \forall x \in \mathbb{R}^{n} \backslash\{\mathbf{0}\} .
\end{aligned}
$$

Then, the system (2) for $\mathcal{S}=\mathcal{S}_{\text {average }}[\tau]$ is almost globally stable for any

$$
\tau>\tau_{\text {ave }}:=\frac{1}{\kappa} \min _{\beta_{1}, \ldots, \beta_{D} \in \mathbb{R}_{>0}} \max _{p, m \in\{1,2, \ldots, D\}} \ln \left(\frac{\beta_{p}}{\beta_{m}} c_{p m}\right) .
$$

It can be seen that the assumptions of Theorem 2 implies the assumptions of Theorem 1 with the same constants $c_{p m}$ and with $\kappa \equiv \kappa_{p}$. Therefore, for a given switched system, the average dwell time found by (10) is larger than or equal to the minimum dwell time found by (6). This is in unison with the fact that the average dwell time condition is a relaxation of the dwell time condition.

Remark 1: Note that the expression of $\tau_{\text {ave }}$ in (6) is equivalent to the so-called maximum cycle mean of weighted digraphs [26] for which fast algorithms exist [25] ${ }^{1}$. More precisely, let $\mathcal{G}=\{\mathcal{V}, \mathcal{E}, \omega\}$ be a weighted digraph where $\mathcal{V}:=\{1, \ldots, D\}$ is the set of vertices, $\mathcal{E} \subsetneq \mathcal{V} \times \mathcal{V}$ is the set of directed edges and $\omega: \mathcal{E} \rightarrow \mathbb{R}$ is the weight function defined as $\omega((i, j))=\ln c_{i j}$ (see Figure III for examples). For a cycle $C=\left\{\left(v_{0}, v_{1}\right), \ldots,\left(v_{L-1}, v_{L}=v_{0}\right)\right\}$ of length $L$, the cycle mean is defined as

$$
\nu(C):=\frac{\sum_{i=0}^{L-1} \omega\left(\left(v_{i}, v_{i+1}\right)\right)}{L} .
$$

Let $\mathcal{C}$ be the set of all simple cycles in $\mathcal{G}$. The maximum cycle mean of $\mathcal{G}$ is defined as

$$
\nu_{\max }=\max _{C \in \mathcal{C}} \nu(C)
$$

which is equal to

$$
\min _{\beta_{1}, \ldots, \beta_{N} \in \mathbb{R}_{>0}} \max _{p, m \in\{1,2, \ldots, D\}} \ln \left(\frac{\beta_{p}}{\beta_{m}} c_{p m}\right)
$$

by [26, Theorem 1.1]. Hence, we have

$$
\tau_{\mathrm{ave}}=\frac{\nu_{\max }}{\kappa} \text {. }
$$

In particular, for bimodal systems, the average dwell time condition (6) can be written as

$$
\tau>\tau_{\text {ave }}:=\frac{\ln c_{12}+\ln c_{21}}{2 \kappa} .
$$

Remark 2: It is straightforward to extend the results in Theorem 1 and Theorem 2 to the case where switchings between subsystems are subject to a given digraph. In this case, we consider the same digraph $\mathcal{G}=\{\mathcal{V}, \mathcal{E}, \omega\}$ as in

\footnotetext{
${ }^{1}$ This also proves that the minimum in (6) is attained.
} 
Remark 1 with a restricted set of edges $\mathcal{E} \subsetneq \mathcal{V} \times \mathcal{V}$. Consequently, $\tau_{\text {ave }}$ can be calculated by (11) with $\nu_{\max }$ computed for the restrcted digraph $\mathcal{G}$.

Example 1: Consider a switched system with $D=3$ with subsystems given as follows:

$$
\begin{aligned}
& f_{1}\left(x_{1}, x_{2}\right)=\left(\begin{array}{c}
-0.1 x_{1}+x_{2}+3 x_{1} x_{2} \\
-x_{1}-0.1 x_{2}-2 x_{1}^{2}+x_{2}^{2}
\end{array}\right) \\
& f_{2}\left(x_{1}, x_{2}\right)=\left(\begin{array}{c}
-0.1 x_{1}+2 x_{2}-0.5 x_{1}^{2}+4 x_{2}^{2} \\
-0.5 x_{1}-0.1 x_{2}-1.5 x_{1} x_{2}
\end{array}\right) \\
& f_{3}\left(x_{1}, x_{2}\right)=\left(\begin{array}{c}
-0.1 x_{1}+0.5 x_{2}-1.5 x_{1} x_{2} \\
-2 x_{1}-0.1 x_{2}+4 x_{1}^{2}-0.5 x_{2}^{2}
\end{array}\right)
\end{aligned}
$$

Assume that $\rho_{1}\left(x_{1}, x_{2}\right)=\left(x_{1}^{2}+x_{2}^{2}\right)^{-5 / 2}, \rho_{2}\left(x_{1}, x_{2}\right)=$ $\left(\left(0.5 x_{1}\right)^{2}+x_{2}^{2}\right)^{-5 / 2}$ and $\rho_{3}\left(x_{1}, x_{2}\right)=\left(x_{1}^{2}+\left(0.5 x_{2}\right)^{2}\right)^{-5 / 2}$. It can be obtained that $\nabla \cdot\left(\rho_{i} f_{i}\right)=\kappa \rho_{i}$ for $i=1,2,3$, where $\kappa=0.3$. Also, it can be calculated that $c_{12}=c_{13}=1$ and $c_{21}=c_{23}=c_{31}=c_{32}=2^{5}$. In the fully connected graph (see $\mathcal{G}_{1}$ in Figure III), then there are five simple cycles: $\mathcal{C}_{(12)}, \mathcal{C}_{(13)}, \mathcal{C}_{(23)}, \mathcal{C}_{(123)}$ and $\mathcal{C}_{(321)}$. Corresponding cycle means are $\nu\left(C_{(12)}\right)=\nu\left(C_{(13)}\right)=\frac{\ln 2^{5}}{2}, \nu\left(C_{(23)}\right)=$ $\ln 2^{5}$, and $\nu\left(C_{(123)}\right)=\nu\left(C_{(321)}\right)=\frac{2 \ln 2^{5}}{3}$. Assume the standard case where switchings are possible between any pair of subsystems, namely we consider the digraph $\mathcal{G}_{1}$ in Figure III. Then, the system is almost globally stable if $\tau>\frac{\nu_{\max }\left(\mathcal{G}_{1}\right)}{\kappa}=\frac{\ln 2^{5}}{0.3}$. On the other hand, if switchings between the subsystem 2 and 3 are not allowed, namely if switchings are subject to the digraph $\mathcal{G}_{2}$ in Figure III, then the system is almost globally stable for $\tau>\frac{\nu_{\max }\left(\mathcal{G}_{2}\right)}{\kappa}=\frac{\ln 2^{5}}{0.6}$.

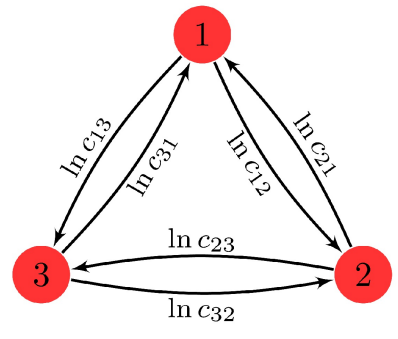

$\mathcal{G}_{1}$

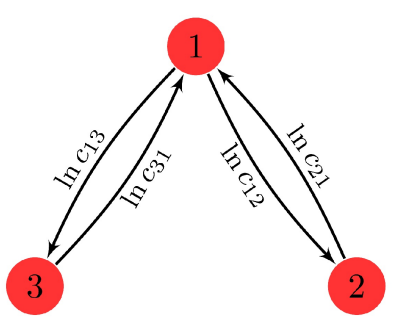

$\mathcal{G}_{2}$
Fig. 1. Weighted digraphs considered in Example 1. Nodes represent subsystems, edges represent admissible swithcings between subsystems and weights are used to calculated the average dwell time.

\section{Proof of the Main Result}

Global transfer operators of nonlinear systems, namely the Frobenius-Perron operator and the Koopman operator will be used for the proof of the main result. These operators are defined on $\mathcal{M}\left(\mathbb{R}^{n}\right)$, the set of equivalence classes of measurable functions where two functions are assumed to be equal if they agree on a set of full Lebesgue measure.

Let us consider first the autonomous system (1) and assume that almost all solutions exist in forward time, which can be ensured, for instance, by the assumptions of Proposition 1. In this case, we can consider the semigroup of Frobenius-Perron operators $\left\{P^{(t)}\right\}_{t \geq 0}$ defined on $\mathcal{M}\left(\mathbb{R}^{n}\right)$ uniquely via

$$
\int_{V} P^{(t)} \rho(x) d x=\int_{\Phi_{t}^{-1}(V)} \rho(x) d x,
$$

where $\Phi_{t}, t \geq 0$ denote time- $t$ solution maps of (1), $V$ is an arbitrary measurable set and $\Phi_{t}^{-1}(V)$ denotes the inverse image of $V$ under the map $\Phi_{t}$. Similarly, the semigroup of Koopman operators $\left\{U^{(t)}\right\}_{t \geq 0}$ can be defined on $\mathcal{M}\left(\mathbb{R}^{n}\right)$ via

$$
U^{(t)} g(x)=g\left(\Phi_{t}(x)\right), \quad t \geq 0 .
$$

Let us define $\langle g, \rho\rangle:=\int_{\mathbb{R}^{n}} g \rho d x$. Duality between $P^{(t)}$ and $U^{(t)}$ is expressed by $\left\langle U^{(t)} g, \rho\right\rangle=\left\langle g, P^{(t)} \rho\right\rangle$. Note that we allow both sides of the duality equation to be infinite (see [27], [28] for more details on transfer operators).

Consider now the switched system (2) and assume that, for any $\sigma \in \mathcal{S}$, almost all solutions exist in forward time. For a switching signal $\sigma(t)$, the semigroup of FrobeniusPerron operators $\left\{P^{(t)}(\sigma)\right\}_{t \geq 0}$ and the semigroup of Koopman operators $\left\{U^{(t)}(\sigma)\right\}_{t \geq 0}$ can be defined as above. For $t \in\left[t_{N}, t_{N+1}\right), P^{(t)}(\sigma)$ and $U^{(t)}(\sigma)$ satisfy

$$
\begin{aligned}
& P^{(t)}(\sigma)=P_{p_{N+1}}^{\left(t-t_{N}\right)} \cdots P_{p_{2}}^{\left(\Delta t_{2}\right)} P_{p_{1}}^{\left(\Delta t_{1}\right)}, \\
& U^{(t)}(\sigma)=U_{p_{1}}^{\left(\Delta t_{1}\right)} U_{p_{2}}^{\left(\Delta t_{2}\right)} \cdots U_{p_{N+1}}^{\left(t-t_{N}\right)},
\end{aligned}
$$

where $P_{i}^{(t)}$ and $U_{i}^{(t)}$ are Frobenius-Perron and Koopman operators of the $i$ 'th subsystem, respectively. Let $B(\varepsilon):=$ $\left\{x \in \mathbb{R}^{n} \mid\|x\|<\varepsilon\right\}$ and $1_{V}$ denote the characteristic function of $V$.

Lemma 1: Assume that almost all solutions of the switched system (2) exist in forward time for all $\sigma \in \mathcal{S}$. Then (2) is almost globally stable if and only if, for each $\sigma \in \mathcal{S}, \int_{0}^{\infty} U^{(\tau)}(\sigma) 1_{B(\varepsilon)^{c}}(x) d \tau<\infty$ for almost every $x$, $\forall \varepsilon>0$.

Proof: (Sufficiency) Define $\quad \mu_{x}(E) \quad:=$ $\int_{0}^{\infty} U^{(\tau)}(\sigma) 1_{E}(x) d \tau$. The (occupation) measure $\mu_{x}(E)$ provides the length of the time that the solution curve $\Phi_{t}(x)$ starting at $x$ stays in the set $E$. Here, proof by contradiction is used to show that the contrapositive statement for a fixed $x$ is true, i.e., $\mu_{x}\left(B(\varepsilon)^{c}\right)=\infty$ for some $\varepsilon>0$ if $\lim _{t \rightarrow \infty} \Phi_{t}(x) \neq 0$. Assume that $\forall \epsilon>0$, $\mu_{x}\left(B(\varepsilon)^{c}\right)<\infty$ and $\lim _{t \rightarrow \infty} \Phi_{t}(x) \neq 0$. Then, by the second assumption there exists $\varepsilon$ such that for all $t>0$, there exists $T(t)>t$ such that $\Phi_{t}(x) \in B(\varepsilon)^{c}$. Furthermore, for all $T$ such that $\Phi_{T}(x) \in B(\varepsilon)^{c}$, there exists $T^{\prime}>T$ such that $\Phi_{T^{\prime}}(x) \in B(\varepsilon / 2)$. If there is no such $T^{\prime}$, then $\Phi_{t}(x) \in B(\varepsilon / 2)^{c}$ for all $t>T$. Hence, $\mu_{x}\left(B_{\varepsilon / 2}^{c}\right)=\infty$, which is a contradiction. Subsequently, construct the sequences $\left\{t_{k}\right\},\left\{t_{k}^{\prime}\right\} \rightarrow \infty$ such that $t_{k+1}^{\prime}>t_{k+1}>t_{k}^{\prime}>t_{k}$ and $\Phi_{x}\left(t_{k}\right) \in B(\varepsilon / 2), \Phi_{x}\left(t_{k}^{\prime}\right) \in B(\varepsilon)^{c}$. With the help of continuity of the solutions, we can construct the following sequences $\left\{\bar{t}_{k}\right\},\left\{\underline{t}_{k}\right\} \rightarrow \infty$ such that $t_{k}^{\prime}>\bar{t}_{k}>\underline{t}_{k}>t_{k}$, $\Phi_{t}(x) \in B(\varepsilon) \backslash B(\varepsilon / 2)$ for $t \in\left(\underline{t}_{k}, \bar{t}_{k}\right)$ and $\left\|\Phi_{\underline{t}_{k}}(x)\right\|=\varepsilon / 2$, $\left\|\Phi_{\bar{t}_{k}}(x)\right\|=\varepsilon$. Due to the local Lipschitz property of $f_{i}(x)$, there is a common Lipschitz constant in $B(\varepsilon)^{c}$. Therefore, $\liminf \operatorname{in}_{k}\left(\bar{t}_{k}-\underline{t}_{k}\right)>0$. As a consequence, $\mu_{x}\left(B(\varepsilon / 2)^{c}\right) \geq \mu_{x}(B(\varepsilon) \backslash B(\varepsilon / 2)) \geq \sum_{k=0}^{\infty}\left(\bar{t}_{k}-\underline{t}_{k}\right)=\infty$, 
which is a contradiction. This proves that $\lim _{t \rightarrow \infty} \Phi_{t}(x)=0$ if $\mu_{x}\left(B(\varepsilon)^{c}\right)<\infty$ for every $\varepsilon>0$. Consider a sequence $\left\{\varepsilon_{n}>0\right\} \rightarrow 0$. For every $\varepsilon_{n}$, define the sets $N_{\varepsilon_{n}}:=$ $\left\{x \mid \mu_{x}\left(B\left(\varepsilon_{n}\right)^{c}\right)=\infty\right\}$ and $N:=\cup_{n} N_{\varepsilon_{n}} . N$ has zero measure, since it is countable union of zero measure sets. The set $S=\left\{x \mid \mu_{x}\left(B(\varepsilon)^{c}\right)=\infty\right.$, for some $\left.\varepsilon>0\right\} \subset N$, since for any $\varepsilon>0$ there exists a sufficiently large $n$ such that $N \supset N_{\varepsilon_{n}} \supset N_{\varepsilon}$. Thus, $S$ has zero measure. Hence, $\lim _{t \rightarrow \infty} \Phi_{t}(x) \neq 0$ for almost every $x$.

(Necessity) If the switched system (2) is almost globally stable, then $\lim _{t \rightarrow \infty} \Phi_{t}(x)=0$ for almost everywhere $x$. Then, for every $\varepsilon>0$, for almost every $x$ there exists $T(x)$ such that $\Phi_{t}(x) \in B(\varepsilon)$ for all $t>T(x)$. Therefore, $\mu_{x}\left(B(\varepsilon)^{c}\right) \leq T(x)$. This implies that, for every $\varepsilon>0$, $\mu_{x}\left(B(\varepsilon)^{c}\right)<\infty$ for almost every $x$.

Lemma 2: The switched system (2) is almost globally stable if, for every $\sigma(t) \in \mathcal{S}$, there exists an almost everywhere positive function $\rho \in \mathcal{M}\left(\mathbb{R}^{n}\right)$ such that

$$
\bar{\rho}:=\int_{0}^{\infty} P^{\sigma}(\tau) \rho d \tau
$$

is integrable on $B_{\varepsilon}^{c}$, for every $\varepsilon>0$

Proof: Using duality and Tonelli's theorem with Lebesgue measure on time variable,

$$
\begin{aligned}
\left\langle\bar{\rho}, 1_{B_{\epsilon}^{c}}\right\rangle & =\int_{0}^{\infty}\left\langle P^{\sigma}(\tau) \rho, 1_{B_{\epsilon}^{c}}\right\rangle d \tau \\
& =\int_{0}^{\infty}\left\langle\rho, U^{(\tau)}(\sigma) 1_{B(\varepsilon)^{c}}(x)\right\rangle d \tau \\
& =\left\langle\rho, \int_{0}^{\infty} U^{(\tau)}(\sigma) 1_{B(\varepsilon)^{c}}(x) d \tau\right\rangle<\infty
\end{aligned}
$$

Since $\rho$ is positive almost everywhere, $\int_{0}^{\infty} U^{\tau} 1_{B(\varepsilon)^{c}}(x) d \tau<$ $\infty$ for almost every $x$.

Lemma 3 ([24]): For a continuously differentiable vector field $f: \mathbb{R}^{n} \rightarrow \mathbb{R}^{n}$ with $f(\mathbf{0})=\mathbf{0}$, suppose that almost all solutions of $\dot{x}=f(x)$ exist for all $t>0$. Assume that there exist a constant $\kappa>0$ and a non-negative, continuously differentiable function $\rho: \mathbb{R}^{n} \backslash\{\mathbf{0}\} \rightarrow \mathbb{R}$ such that

- $\rho(x)$ is integrable away from $\mathbf{0}$, and

- $\nabla \cdot(\rho f) \geq \kappa \rho$.

Then, for all $t>0$,

$$
P^{(t)} \rho \leq \mathrm{e}^{-\kappa \mathrm{t}} \rho .
$$

We can now state the proof of the main result.

Proof: [Theorem 2] Define the index of the subsystem which is active for $t \in\left[t_{k-1}, t_{k}\right)$ as $p_{k}$ and time duration of $k$ th switching as $\Delta t_{k}=t_{k}-t_{k-1}$ for $k \geq 1$. For simplicity, assume that $t_{0}=0$. Using Lemma 3 and assumptions in Theorem 2, for $t \in\left[t_{N}, t_{N+1}\right)$

$$
\begin{aligned}
P^{(t)}(\sigma) \rho_{p_{1}} & =P_{p_{N+1}}^{\left(t-t_{N}\right)} \cdots P_{p_{2}}^{\left(\Delta t_{2}\right)} P_{p_{1}}^{\left(\Delta t_{1}\right)} \rho_{p_{1}} \\
& \leq P_{p_{N+1}}^{\left(t-t_{N}\right)} \cdots P_{p_{2}}^{\left(\Delta t_{2}\right)} e^{-\kappa \Delta t_{1}} \rho_{p_{1}} \\
& \leq P_{p_{N+1}}^{\left(t-t_{N}\right)} \cdots P_{p_{2}}^{\left(\Delta t_{2}\right)} e^{-\kappa \Delta t_{1}+\ln c_{p_{1} p_{2}} \rho_{p_{2}}} \\
& \leq e^{-\kappa t} e^{\sum_{i=1}^{N} \ln c_{p_{i} p_{i+1}} \rho_{\max }}
\end{aligned}
$$

where $\rho_{\max }(x)=\max _{i \in\{1,2, \ldots, D\}} \rho_{i}(x)$. Consequently, we have

$$
\int_{0}^{\infty} P^{(t)}(\sigma) \rho_{p_{1}} d t \leq \int_{0}^{\infty} e^{-\kappa t} e^{\sum_{i=1}^{N(t)} \ln c_{p_{i} p_{i+1}} \rho_{\max } d t},
$$

where $N(t)$ is the number of switchings before time $t$. Since $\rho_{\max }$ is independent of $t$ and $N(t)$ is constant for $t \in\left[t_{N}, t_{N+1}\right)$, we obtain

$\int_{0}^{\infty} P^{(t)}(\sigma) \rho_{p_{1}} d t \leq \rho_{\max } \sum_{N=0}^{\infty} e^{\sum_{i=1}^{N} \ln c_{p_{i} p_{i+1}}} \int_{t_{N}}^{t_{N+1}} e^{-\kappa t} d t$

After replacing the upper bound of the last integral by infinity, we have

$$
\begin{aligned}
\int_{0}^{\infty} P^{(t)}(\sigma) \rho_{p_{1}} d t & \leq \frac{\rho_{\max }}{\kappa} \sum_{N=0}^{\infty} e^{-\kappa t_{N}+\sum_{i=1}^{N} \ln c_{p_{i} p_{i+1}}} \\
& =\frac{\rho_{\max }}{\kappa} \sum_{N=0}^{\infty} e^{a_{N}}
\end{aligned}
$$

where $a_{N}=-\kappa t_{N}+\sum_{i=0}^{N} \ln c_{p_{i} p_{i+1}}$. Now, we will show that the sum in (13) is finite, if the average dwell time satisfies

$$
\tau>\bar{\tau}_{\text {ave }}=\frac{1}{\kappa} \max _{p, m \in\{1,2, \ldots, D\}} \ln c_{p m} .
$$

Using the definition of the average dwell time, we have

$$
\begin{aligned}
a_{N} & \leq-\kappa\left(N-N_{0}\right) \tau+\sum_{i=0}^{N} \ln c_{p_{i} p_{i+1}} \\
& =\kappa \tau N_{0}+-\kappa \sum_{i=0}^{N}\left(\tau-\frac{\ln c_{p_{i} p_{i+1}}}{\kappa}\right) \\
& \leq \kappa \tau N_{0}-\kappa N\left(\tau-\bar{\tau}_{\text {ave }}\right)
\end{aligned}
$$

Finally, we have

$$
\begin{aligned}
\int_{0}^{\infty} P^{(t)}(\sigma) \rho_{p_{1}} d t & \leq \frac{\rho_{\max }}{\kappa} \sum_{N=0}^{\infty} e^{\kappa \tau N_{0}-\kappa N\left(\tau-\bar{\tau}_{\text {ave }}\right)} \\
& \leq \frac{\rho_{\max }}{\kappa} A
\end{aligned}
$$

where $A=e^{\kappa \tau N_{0}} /\left(1-e^{-\kappa\left(\tau-\bar{\tau}_{\text {ave }}\right)}\right)$. Since the integral on the left-hand side is finite, the switched system with $\tau>\bar{\tau}_{\text {ave }}$ is stable by Lemma 2 .

The average dwell time condition can be relaxed by defining new Lyapunov densities $\overline{\rho_{i}}=\beta_{i} \rho_{i}$ for each subsystem, where $\beta_{i}>0$. After this transformation, new compatibility conditions are satisfied with $\overline{c_{i j}}=\frac{\beta_{i}}{\beta_{j}} c_{i j}$. Since $\beta_{i}$ 's are arbitrary, they can be chosen so as to minimize $\bar{\tau}_{\text {ave }}$ in (14), which concludes the proof.

\section{CONClusion AND Discussion}

A sufficient condition for the almost global stability of nonlinear switched systems with average dwell time has been presented. The proof leans upon the properties of two global transfer operator of nonlinear systems: the Frobenius-Perron and the Koopman operators. We have shown that the average 
dwell time corresponds to maximum cycle mean of weighted digraphs for which there exist efficient algorithms.

Two possible directions of research can be considered. The first one is a controller design algorithm for systems with the average dwell time restrictions, which can be based on the almost global stabilization of nonlinear switched control systems. The second direction is the study of other restricted classes of switching signals, such as switching signals with mode-dependent [29] and edge-dependent [30] average dwell time.

\section{REFERENCES}

[1] D. Angeli, "An almost global notion of input-to-state stability," IEEE Transactions on Automatic Control, vol. 49, no. 6, pp. 866-874, JUN 2004.

[2] S. Prajna, P. A. Parrilo, and A. Rantzer, "Nonlinear control synthesis by convex optimization," IEEE Transactions on Automatic Control, vol. 49, no. 2, pp. 310-314, 2004.

[3] A. Rantzer, "A dual to Lyapunov's stability theorem," Systems \& Control Letters, vol. 42, pp. 1-17, 2001.

[4] P. Monzon, "Almost global attraction in planar systems," Systems \& Control Letters, vol. 54, pp. 753-758, 2005.

[5] — , "Almost global stability of dynamical systems," Ph.D. dissertation, Udelar, Uruguay, 2006.

[6] U. Vaidya and P. G. Mehta, "Lyapunov measure for almost everywhere stability," IEEE Transactions on Automatic Control, vol. 53, no. 1, pp. 307-323, 2008.

[7] R. Potrie and P. Monzon, "Local implications of almost global stability," Dynamical Systems, vol. 24, no. 1, pp. 109-115, 2009.

[8] V. Grushkovskaya and A. Zuyev, "Attractors of nonlinear dynamical systems with a weakly monotonic measure," Journal of Mathematical Analysis and Applications, vol. 422, pp. 559-570, 2015.

[9] R. Rajaram, U. Vaidya, M. Fardad, and B. Ganapathysubramanian, "Stability in the almost everywhere sense: A linear transfer operator approach," Journal of Mathematical Analysis and Applications, vol. 368, no. 1, pp. 144-156, 2010.

[10] R. Rajaram and U. Vaidya, "Lyapunov density for coupled systems," Applicable Analysis, vol. 94, no. 1, pp. 169-183, 2015.

[11] Ö. Karabacak, R. Wisniewski, and J. Leth, "On the almost global stability of invariant sets," in Proceedings of the 2018 European Control Conference, 2018.

[12] Ö. Karabacak, R. Wisniewski, and A. Kıvilcım, "Almost global finite-time stability of invariant sets," in Proceedings of 57th IEEE Conference on Decision and Control, 2018.

[13] A. Rantzer and F. Ceragioli, "Smooth blending of nonlinear controllers using density functions," in Proceedings of the 2001 European Control Conference, 2001, pp. 2851-2853.

[14] U. Vaidya, P. G. Mehta, and U. V. Shanbhag, "Nonlinear stabilization via control lyapunov measure," IEEE Transactions on Automatic Control, vol. 55, no. 6, pp. 1314-1328, 2010.

[15] U. Vaidya and C. Venkatesh, "Computation of the Lyapunov measure for almost everywhere stochastic stability," in Proceedings of the IEEE Conference on Decision and Control, 2015, pp. 7042-7047.

[16] A. Ataei and Q. Wang, "Robust nonlinear control design for a hypersonic aircraft using sum of squares method," in ASME 2010 Dynamic Systems and Control Conference (DSCC 2010), vol. 1, 01 2010.

[17] M. Seo, H. Kwon, and H. Choi, "Nonlinear missile autopilot design using a density function-based sum-of-squares optimization approach," in 2015 IEEE Conference on Control Applications (CCA), Sep. 2015, pp. 947-952.

[18] S. Pak Khesal and I. Mohammadzaman, "Nonlinear robust roll autopilot design using sum-of-squares optimization," Journal of Dynamic Systems, Measurement, and Control, vol. 140, p. 111005, 062018.

[19] S. Prajna and A. Rantzer, "Convex programs for temporal verification of nonlinear dynamical systems," SIAM Journal on Control and Optimization, vol. 46, no. 3, pp. 999-1021, 2007.

[20] I. Masubuchi and Y. Ohta, "Analysis of almost-everywhere stability of a class of discontinuous systems via lyapunov densities," in 2016 European Control Conference (ECC), June 2016, pp. 567-574.
[21] P. Monzón, "Almost global stability of time-varying systems," in Proceedings of the Congresso Brasileiro de Automatica, 2006, pp. 198-201.

[22] I. Masubuchi and T. Kikuchi, "Lyapunov density for almost attraction of nonlinear time-varying systems: A condition without assuming local stability," in 2017 25th Mediterranean Conference on Control and Automation (MED), July 2017, pp. 169-173.

[23] U. Vaidya, "Stochastic stability analysis of discrete-time system using lyapunov measure," in 2015 American Control Conference (ACC), July 2015, pp. 4646-4651.

[24] Ö. Karabacak, A. Kivilcim, and R. Wisniewski, "Almost Global Stability of Nonlinear Switched Systems with Time-Dependent Switching," sep 2018. [Online]. Available: http://www.preprints.org/manuscript/201809.0514/v1

[25] A. Dasdan, "Experimental analysis of the fastest optimum cycle ratio and mean algorithms," ACM Transactions on Design Automation of Electronic Systems, vol. 9, no. 4, pp. 385-418, 2004.

[26] M. Golitschek, "Optimal cycles in doubly weighted graphs and approximation of bivariate functions by univariate ones," Numerische Mathematik, vol. 39, no. 1, pp. 65-84, 1982.

[27] A. Lasota and M. C. Mackey, Chaos, fractals and noise: Stochastic aspects of dynamics. New York: Springer-Verlag, 1994.

[28] E. Çınlar, Probability and Stochastics. Springer, New York, 2001.

[29] X. Zhao, L. Zhang, P. Shi, and M. Liu, "Stability and Stabilization of Switched Linear Systems With Mode-Dependent Average Dwell Time," IEEE Transactions on Automatic Control, vol. 57, no. 7, pp. 1809-1815, JUL 2012.

[30] L. Hou, X. Zhao, H. Sun, and G. Zong, "l(2) - 1(infinity) filtering of discrete-time switched systems via admissible edge-dependent switching signals," SYSTEMS \& CONTROL LETTERS, vol. 113, pp. 17-26, MAR 2018. 